\title{
Interferon action revisited
}

\author{
from Paula M. Pitha
}

INTERFERONS are species-specific cellular glycoproteins synthesised by a wide variety of animal cells in response to viral infection (or various non-viral stimuli) which induce antiviral resistance in homologous cells. In interferontreated cells, a wide variety of different viruses do not replicate well. It was shown with a majority of the viruses studied that the primary effect of interferon is a general inhibition of viral protein synthesis. In addition to this block it has also been found that with some viruses such as VSV and SV40 the transcription of viral mRNA is decreased in interferon-treated cells. Oncornavirus replication seems to be inhibited by interferon at the level of virus maturation and assembly.

The nature of the molecular mechanism by which interferon induces the antiviral state in the cell has not yet been clarified. Interferon by itself is not an antiviral agent. The antiviral state does not develop in enucleated cells or in cells treated with actinomycin D or cycloheximide. This indicates that the antiviral effect of interferon requires both transcription and translation of the cellular genome and leads to the proposal that there is an interferon-induced antiviral protein (Taylor Biochem. biophys. Res. Commun. 14, 447; 1964). The situation, however, is not that simple; recent results from the laboratories of 1 . Kerr (MRC, Mill Hill, London), P. Lengyel (Yale University, Connecticut) and $\mathbf{M}$. Revel (Weizmann Institute of Science, Rehovot) with cell-free protein synthesising systems, indicate that interferon treatment induces the synthesis of several translational inhibitors.

The first step in the induction of the antiviral effect is the interaction of interferon with the cellular membrane of a sensitive cell, and it has been observed that the interferon binding involves both cellular gangliosides and glycoproteins (Besancon \& Ankel Nature 252, 478; 1974; Vengris et al. Virology 72, 486; 1976; Kohn et al. Proc. natn. Acad. Sci. U.S.A. 73, 3695; 1976). The cellular uptake of interferon is not required for the antiviral effect (Ankel et al. Proc. natn. Acad. Sci. U.S.A. 70, 2360; 1973); the sensitivity to interferon seems to be determined by a receptor located at the plasma membrane. Using antiserum to interferon, which neutralised all free inter-

Paula M. Pitha is Associate Professor of Oncology in the Cancer Center of the Johns Hopkins University School of Medicine. feron in the medium, Vengris et al. (Virology 64, 410; 1975) demonstrated that for the development of the antiviral state interferon had to interact with the external cellular membrane of the cell producing interferon.

The results obtained by several groups (for example F. Ruddle at Yale University, Connecticut, and F. C. Chany at INSERM, Paris) using mouse-human cell hybrids demonstrated that the sensitivity to human interferon is governed by human chromosome 21 . Trisomy 21 cells were markedly more sensitive to human interferon than normal diploid cells or monosomy 21 cells (Tan et al. Science 186, 61; 1974; Chany et al. Proc. natn. Acad. Sci. U.S.A. 72, 3129; 1975). Antibodies to hybrid cells containing human chromosome 21 were found to inhibit the antiviral effect of human interferon in human cells. Revel et al. (Nature 260, 139; 1976) suggested that chromosome 21 codes for the receptor for human interferon on the cell surface.

The species specificity of interferons seems to be also determined at the receptor level. Recent data indicate that in primate-mouse hybrid cells containing only one or a few human chromosomes interferon specificity is due to the interaction with the receptor but the antiviral mechanism induced with both mouse and human interferon is of mouse origin (Chany, Biomedicine Express 24, 148; 1976). This indicates that at least some components of the antiviral effect do not have a requirement for species specificity. Samuel and Joklik (Virology 75, 166; 1976) came to a similar conclusion when they showed that interferon treatment induced a ribosome-associated inhibitor of viral mRNA translation in cell-free systems that could be dissociated from ribosomes and inhibit the translation of viral mRNA in a heterologous cellfree protein synthesising system.

In this issue of Nature (page 422) Blalock and Baron from the University of Texas at Galveston present another type of evidence that the interferoninduced antiviral 'machinery' may not be species specific. The authors also suggest that the antiviral compound induced by interferon treatment of the homologous cells can be transferred to the cell of the heterologous species and elicit in it resistance to viral infection. The experiments are based on cocultivation of mouse cells (sensitive to mouse interferon) with interferoninsensitive human cells in the presence of mouse interferon, infection with
VSV or vaccinia virus, and measurement of virus yield. To achieve the effect close contact between the two cell populations was required; the effect was not observed in cultures plated at low cell density. Actinomycin D treatment of the mouse cells blocked both the antiviral effect in the mouse cells and their ability to transfer resistance to the heterologous cells. It seems, therefore, that the transferred compound may be a product of interferon induction rather than interferon itself.

This conclusion, however, is based on experiments done in mass cultures and the challenging viruses replicated both in homologous and heterologous cells. In the future much clearer data would be obtained if the infecting virus could not replicate in the cells homologous to the interferon but only in the cells to which the antiviral compound was transferred. It has been shown previously that the effect of interferon is an all-or-none phenomenon in $\mathrm{L}$ cells and in chick embryo cells (Chang et al. J. gen. Virol. 20, 139; 1973; Radke et al. J. Virol. 13, $623 ; 1974)$, and thus it should be possible to measure the transfer of viral resistance from the cell in the antiviral state to the adjacent neighbour. Fluorescent antibody or infectious centre techniques could be used to confirm the requirement for cell-cell contact.

One of the remarkable properties of the interferon system is its high biological activity. The specific activity of both human and mouse interferon is estimated to be close to $10^{9}$ units per $\mathrm{mg}$ protein (one unit is the amount of interferon which inhibits virus replication by $50 \%$ ). It is probable that such a system involves mechanisms which amplify the initial response. Both the above described transfer of antiviral resistance and the mobility of the interferon receptors on the cell surface (Lebon et al. Proc Soc. exp. Biol. Med. 149, 108; 1975) may be amplification factors in the interferon system.

Currently it seems that the action of interferon at the cellular surface resembles in many respects the action of the polypeptide hormones. The major task for the future is to answer the question of how the impulse from the cell membrane is transferred to the nucleus. One possibility which remains to be determined is that the antiviral effect of interferon is mediated through a second message such as cyclic AMP (Weber \& Stewart J. gen. Virol 28, 363; 1975). 